\title{
Role of the range of the dipole function in the classical dynamics of molecular dissociation
}

\author{
Emanuel F. de Lima, ${ }^{*}$ Tárcius N. Ramos, ${ }^{\dagger}$ and R. Egydio de Carvalho ${ }^{\ddagger}$ \\ Instituto de Geociências e Ciências Exatas, UNESP-Univ Estadual Paulista, Rio Claro, São Paulo 13506-900, Brazil
}

(Received 2 August 2012; revised manuscript received 8 December 2012; published 3 January 2013)

\begin{abstract}
The dissociation dynamics of heteronuclear diatomic molecules induced by infrared laser pulses is investigated within the framework of the classical driven Morse oscillator. The interaction between the molecule and the laser field described in the dipole formulation is given by the product of a time-dependent external field with a position-dependent permanent dipole function. The effects of changing the spatial range of the dipole function in the classical dissociation dynamics of large ensembles of trajectories are studied. Numerical calculations have been performed for distinct amplitudes and carrier frequencies of the external pulses and also for ensembles with different initial energies. It is found that there exist a set of values of the dipole range for which the dissociation probability can be completely suppressed. The dependence of the dissociation on the dipole range is explained through the examination of the Fourier series coefficients of the dipole function in the angle variable of the free system. In particular, the suppression of dissociation corresponds to dipole ranges for which the Fourier coefficients associated with nonlinear resonances are null and the chaotic region in the phase space is reduced to thin layers. In this context, it is shown that the suppression of dissociation of heteronuclear molecules for certain frequencies of the external field is a consequence of the finite range of the corresponding permanent dipole.
\end{abstract}

DOI: 10.1103/PhysRevE.87.014901

PACS number(s): 05.45.Ac, 05.45.Gg, 33.80.Gj

The classical driven Morse oscillator has been extensively applied to the study of the dissociation dynamics of heterodiatomic molecules by means of infrared laser fields [1-12]. The interaction between the molecule and the laser field is commonly given by the product of a time-dependent external field, which accounts for the electric field of the laser, with a position-dependent function, associated with the permanent dipole moment of the molecule. Consequently, the dissociation dynamics depends on both the external field and the dipole function. Understanding the role of the dipole function in the dynamics of the Morse oscillator can help to gain physical insights on the dissociation dynamics of diatomic molecules.

The concept of a dipole function for a diatomic molecule arises within the Born-Oppenheimer picture [13-15]. In this picture, the electronic wavefunction depends parametrically on the internuclear distance for a given molecular state. The dipole moment of the molecular charge distribution can be represented by a function of the internuclear distance. Diatomic molecules possess many forms of dipole functions [16-19], which typically go asymptotically to zero for large internuclear distances. Correspondingly, one can associate an effective spatial range with a dipole function.

The free Morse oscillator has two distinct energy regions. A particle placed in the bound region performs oscillatory motion, which represents the vibration of the molecule. A particle placed in the unbound region escapes to infinity and this scenario represents the separation of the atomic pair. When the oscillator is subjected to an external driving field, a particle in the bound region can escape to the unbound region and this transition corresponds to the dissociation of the molecule. The dissociation occurs through chaotic routes,

\footnotetext{
*eflima@rc.unesp.br

†tarciusnramos@gmail.com

${ }^{\ddagger}$ regydio@rc.unesp.br
}

as a result of the destruction of Kolmogorov-Arnold-Moser (KAM) tori in phase-space for increasing values of the field amplitude [3,20].

Several works have investigated the classical dissociation dynamics within the driven Morse model [1-12,21-26]. Also, many quantum-classical comparisons have been performed and correspondences between the two theories have been found in some situations [27-37]. It has been shown that although the quantum and classical dissociation probabilities do not exactly agree in general, the classical calculations can give good estimates for the dissociation thresholds [29]. In fact, agreement between classical and quantum results is expected whenever the frequency of the external field is not tuned to a resonant multiphoton transition [28,29,33]. Most of the above cited works have considered linear approximation for the dipole function, which corresponds to an interaction with infinite spatial range. However, overtone transitions have been investigated for different forms of the dipole function, and it was found that the nonlinearity of the dipole moment is critical for the excitation to occur in the subpicosecond timescale [25]. In addition, it has been shown that the oscillatory behavior of the dipole function influences the splitting of the separatrix and consequently the dissociation dynamics [26].

The goal of this work is to investigate the effects of the range of the dipole function in the classical dissociation dynamics of heteronuclear diatomic molecules within the driven Morse model. We consider an analytical form for the dipole function with adjustable parameters that allow for the control of the effective range of the dipole. We study the classical dissociation probability for large ensembles of particles as a function of the dipole range.

We describe the classical one-dimensional driven Morse oscillator, modeling the dissociation of a heteronuclear diatomic molecule under an infrared linearly polarized laser field. Only pure vibrational motion on a single electronic state is considered and dimensionless variables are used throughout (see Refs. [5,22]). The Hamiltonian of the system is written in two parts: $H(x, p, t)=H_{0}(x, p)+H_{1}(x, t)$. $H_{0}$ represents 
relative motion of the nuclei of the free molecule,

$$
H_{0}=\frac{p^{2}}{2}+\frac{1}{2}\left(\mathrm{e}^{-2 x}-2 \mathrm{e}^{-x}\right),
$$

with $x$ standing for the internuclear distance and $p$ for its conjugate momentum. A particle is in the bound energy region if its energy $E$ is in the interval $\left[-\frac{1}{2}, 0\right]$, while it is in the unbound region if $E>0$. The two regions are divided by a separatrix at $E=0$.

$H_{1}$ represents the laser-molecule interaction given in the electric dipole approximation as

$$
H_{1}=-\mu(x, \xi) \epsilon(t),
$$

where the time-dependent external field $\epsilon(t)$ represents the laser electric field, which is given by a pulse,

$$
\epsilon(t)=\epsilon_{0} s(t) \sin (\omega t),
$$

acting from $t=0$ to a final time $t=t_{f}$, with carrier frequency $\omega$, amplitude $\epsilon_{0}$, and envelope function $s(t)$.

We introduce the dimensionless dipole function $\mu(x, \xi)$,

$$
\mu(x, \xi)=\left(x+x_{e}\right) \mathrm{e}^{-\xi\left(x+x_{e}\right)^{q}},
$$

where $q, x_{e}$, and $\xi$ are adjustable nonnegative parameters. An effective range $x_{R}$ is associated to $\mu(x, \xi)$ through $x_{R}=$ $\xi^{-1 / q}-x_{e}$. In particular, for fixed $q$ and $x_{e}$, as $\xi \rightarrow 0, x_{R} \rightarrow$ $\infty$, and an infinite-range linear dipole function is obtained, whereas as $\xi \rightarrow \infty, x_{R} \rightarrow 0$, the dipole function tends to zero. With properly chosen parameters, the form given in Eq. (4) can approximate the dipole function of several hydrogen halides [19]. Realistic forms for the dipole function of the hydrogen
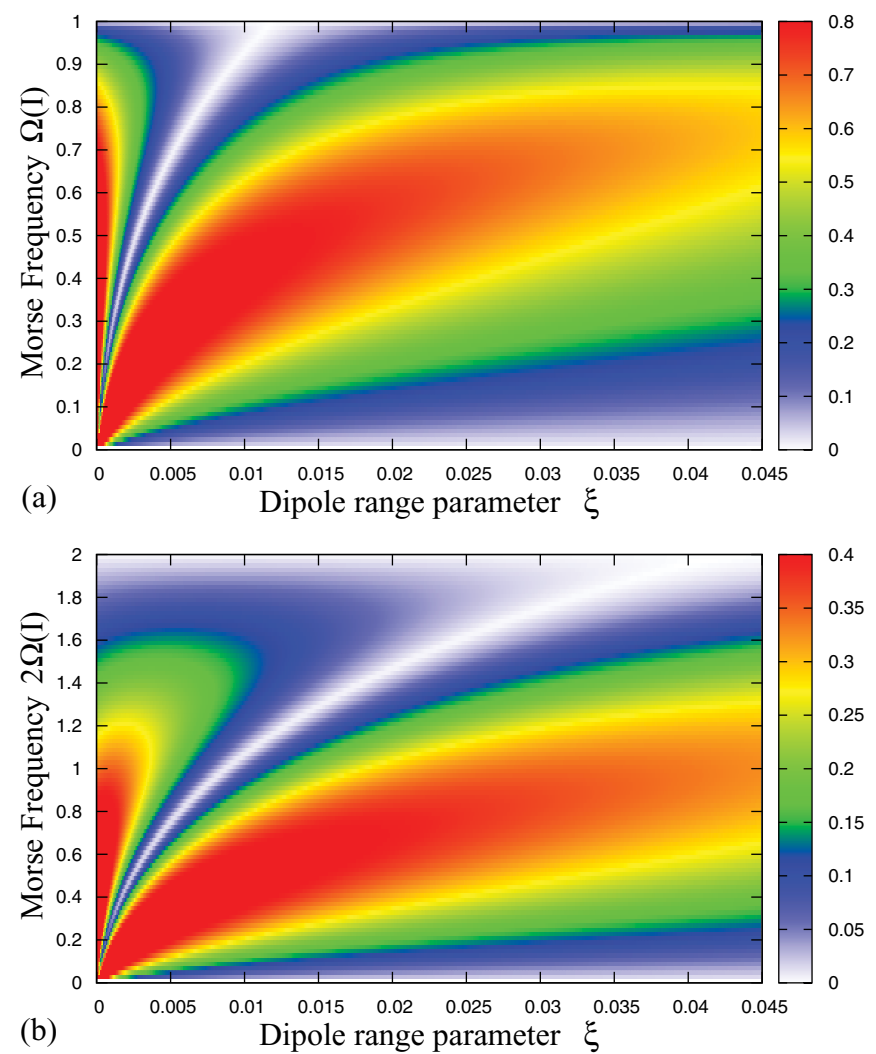

FIG. 1. (Color online) $\left|V_{m}(I, \xi)\right|$ as a function of $\xi$ and $m \Omega(I)$ for (a) $m=1$ and (b) $m=2 . q=4$. fluoride (HF) and for the $\mathrm{OH}$ radical can be obtained with the set of parameters given in Refs. [4,38].

In analogy with quantum mechanics, a classical dissociation probability may also be defined. A large number, $N_{\text {traj }}$, of trajectories is propagated according to Hamilton's equations of motion. The same value of energy is chosen for all trajectories in the initial ensemble, which corresponds to a molecule prepared in a given vibrational energy level. The initial conditions for the calculations are chosen to correspond to high-lying vibrational levels, which can be prepared through selective vibrational excitation [38]. The classical dissociation probability $P_{D}$ is calculated by counting the number $n$ of escaping trajectories for which the energy is greater than the separatrix energy by the end of the external excitation and calculating the fraction

$$
P_{D}=\frac{n}{N_{\text {traj }}} .
$$

In order to analyze the motion near a nonlinear resonance, the canonical variables $(x, p)$ are written in terms of actionangle variables $(\theta, I)$ as [32],

$$
\begin{gathered}
x=\ln \left[\frac{1-\sqrt{1-(1-I)^{2}} \cos (\theta)}{(1-I)^{2}}\right], \\
p=\frac{(1-I) \sqrt{1-(1-I)^{2}} \sin (\theta)}{1-\sqrt{1-(1-I)^{2}} \cos (\theta)} .
\end{gathered}
$$
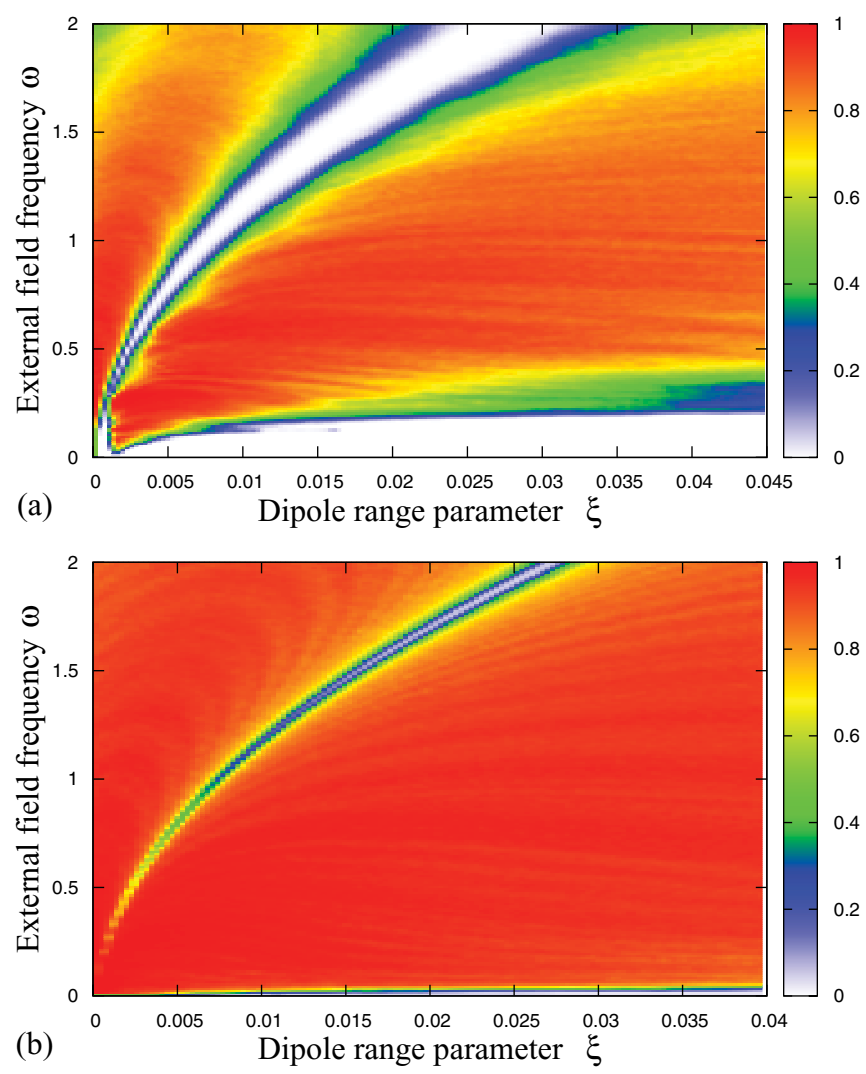

FIG. 2. (Color online) $P_{D}$ as a function of $\xi$ and $\omega$ for the initial action (a) $I(t=0)=0.7$ and (b) $I(t=0)=0.927 . q=4$ and $\epsilon_{0}=0.02$. 

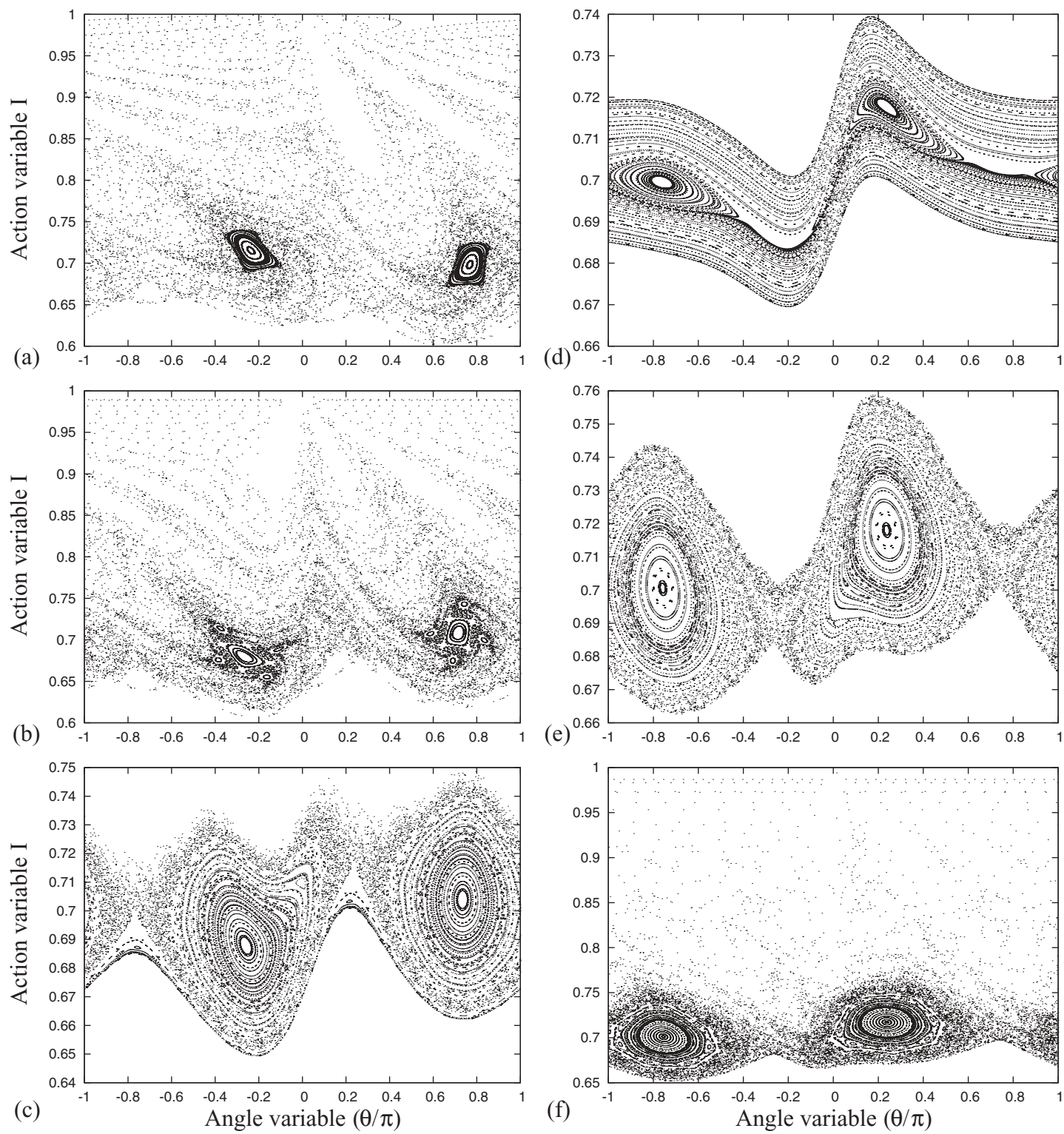

FIG. 3. Stroboscopic plots for (a) $\xi=0\left(x_{R}=\infty\right)$, (b) $\xi=0.002\left(x_{R}=2.6\right)$, (c) $\xi=0.0024\left(x_{R}=2.4\right)$, (d) $\xi=\xi_{2}^{*}=0.0033\left(x_{R}=2\right)$, (e) $\xi=0.004\left(x_{R}=1.8\right)$, and (f) $\xi=0.005\left(x_{R}=1.6\right) . I(t=0)=0.7, q=4, \epsilon_{0}=0.005$, and $\omega=2 \Omega(I=0.7)$.

The total Hamiltonian can be expressed as $H(\theta, I, t)=$ $H_{0}(I)-\mu(\theta, I, \xi) \epsilon(t)$, where the free molecule Hamiltonian is written only in terms of the action variable as $H_{0}(I)=$ $-\frac{1}{2}(1-I)^{2}$. Writing the dipole function as a Fourier series in the angle variable, we have

$$
\begin{aligned}
H(\theta, I, t)= & H_{0}(I)-\frac{1}{2} \epsilon_{0} s(t) \sum_{n=0}^{\infty} V_{n}(I, \xi)[\sin (\omega t-n \theta) \\
& +\sin (\omega t+n \theta)]
\end{aligned}
$$

where the Fourier coefficients $V_{n}(I, \xi)$ are given by

$$
\begin{aligned}
& V_{0}(I, \xi)=\frac{1}{\pi} \int_{0}^{\pi} \mu(\theta, I, \xi) d \theta . \\
& V_{n}(I, \xi)=\frac{2}{\pi} \int_{0}^{\pi} \mu(\theta, I, \xi) \cos (n \theta) d \theta, \quad n=1,2, \ldots
\end{aligned}
$$

An isolated nonlinear resonance is defined by

$$
\omega=n \Omega\left(I_{n}\right)=n\left(1-I_{n}\right)
$$

where $n$ is an integer and $\Omega(I)=\partial H_{0} / \partial I$ is the frequency of the unperturbed motion. In the perturbation theory framework, the most relevant terms are those which satisfy the resonance condition expressed in Eq. (10). Due to its finite-time duration, the external field has a finite bandwidth in the frequency domain $\Delta \omega$. Suppose that for a given carrier frequency $\omega, m$ is the integer that fulfill the resonance condition for some value of the action variable $I_{m}$, i.e., $\omega=m \Omega\left(I_{m}\right)$. The resonance condition is also satisfied for an interval of action $\Delta I_{\omega}$ centered at $I_{m}$, such that $|\Delta \omega|=m\left|\Delta I_{\omega}\right|$. Assume that at $t=0, I(0) \approx I_{m}$ and that the value of the action does not depart too much from its initial value as the system evolves. If we further assume that the interval between this resonance and the nearest resonance is greater than $\Delta I_{\omega}$, i.e., 
$I_{m+1}-I_{m}=\Omega\left(I_{m+1}\right) / m>\Delta I_{\omega}$, we may retain only the term in the vicinity of the $m$ resonance and neglect fast oscillatory terms,

$$
H(\theta, I, t) \approx H_{0}(I)-\frac{1}{2} \epsilon_{0} s(t) V_{m}(I, \xi) \sin (\omega t-m \theta) .
$$

The square root of $\epsilon_{0}\left|V_{m}(I, \xi)\right|$ is proportional to the width of the corresponding resonance island and, according to the Chirikov picture [20], the onset of the chaotic dissociation is related to the overlap of neighboring resonance islands. Therefore, large values of the Fourier coefficients $V_{m}(I, \xi)$ favor dissociation, whereas small values of $V_{m}(I, \xi)$ do not. In Fig. 1, the absolute values of the Fourier coefficients are shown for $m=1,2$ in Figs. 1(a) and 1(b), respectively, as a function of $\xi$ and of $m \Omega(I) .\left|V_{m}(I, \xi)\right|$ is close to zero for some values of $m \Omega(I)$ and $\xi$, indicated by the white regions in the plot. It should be noticed that for increasing frequency bandwidths and amplitudes of the external field, additional Fourier components have to be taken into account in the approximation made in Eq. (11).

The number of trajectories used in the calculations was $N_{\text {traj }}=10^{3}$. No appreciable change in $P_{D}$ has been observed for larger ensembles of trajectories. The ensemble of initial conditions was uniformly distributed over the angle variable $\theta$ for a given value of the action. The duration of the interaction is fixed to $t_{f}=785$, which corresponds to a time of 1 ps for the parameters of HF, while $x_{e}=2.135$. Two types of envelope function were considered: square, $s_{\mathrm{sq}}(t)=1$, and sine-squared, $s_{\sin }(t)=\sin ^{2}\left(\pi t / t_{f}\right)$. While the use of square envelopes allows for the investigation of the system dynamics by means of stroboscopic maps, the use of sine-squared envelopes represents a more realistic time dependence of the interaction Hamiltonian. The frequency bandwidths are $\Delta \omega \approx 0.01$ and $\Delta \omega \approx 0.016$ for the square and sine-squared pulses, respectively.

The panels of Fig. 2 show the dissociation probability as a function of the range parameter and of the frequency of the external field for the initial action of the ensemble equal to 0.7 [Fig. 2(a)] and 0.927 [Fig. 2(b)] for fixed amplitude of the field and square envelope. In Fig. 2(a), $P_{D}$ is close to zero for some values of $\xi$ and $\omega$ corresponding to the white regions in the plot. It is remarkable that for fixed range parameter $\xi$ there are high-frequency values $(\omega>1)$ for which the dissociation vanishes. In Fig. 2(b), the region of negligible dissociation still remains but it is considerably smaller due to the fact that the initial energy of the ensemble is very close to the separatrix. The qualitative resemblance between these panels and the panels in Fig. 1 is apparent, indicating that the Fourier coefficients contain important information about the dissociation dynamics.

Figure 3 shows stroboscopic plots for six values of $\xi$ for the external pulse with a square envelope. The panels were constructed plotting the action-angle variables $(I, \theta)$ at each period of the external field $2 \pi / \omega$ for an ensemble starting with action $I(t=0)=0.7$. The trajectories are plotted only while they are in the bound energy region. The field amplitude was set to $\epsilon_{0}=0.005$ and the external field frequency was chosen such that $\omega=2 \Omega(I=0.7)=0.6$. Figure 3(a) shows the case corresponding to $\xi=0$. In this case, $P_{D}=0.86$ and a large chaotic sea is observed along with two primary resonant islands. For $\xi=0.002$ [Fig. 3(b)], $P_{D}=0.44$ and the two resonance islands are surrounded by four secondary islands, while the phase space is dominated by the chaotic region. For $\xi=0.0024, P_{D}=0.025$ and the regular regions surrounding the resonance islands have increased. In Fig. 3(d), $\xi=\xi_{2}^{*}=0.0033$ and no dissociation occurs. The phase space is considerably regular, with all trajectories confined between KAM curves, which envelope two resonance islands. For $\xi=0.004, P_{D}=0.097$ and the phase space resembles that of Fig. 3(c) with two large resonance islands involved by localized chaotic regions. For $\xi=0.005$, the dissociation probability increases to 0.385 . The effective range $x_{R}$ in Figs. 3(b)-3(f) are, respectively, 2.6, 2.4, 2, 1.8, and 1.6.

As an application, we consider the specific case of the $\mathrm{HF}$ molecule. The values of the parameters are set to $\xi_{\mathrm{HF}}=$ $0.0029, x_{e}=2.135$, and $q=4$, which implies an effective range $x_{R}=2.17(\approx 3.5$ in atomic units $)$. The action $I=0.7$ corresponds to the vibrational bound level $v=15$. Figure 4(a) shows the dissociation probability under square and sinesquared infrared pulses as a function of $\omega$. The panel also presents the results for $\xi=0$. The amplitude is fixed to

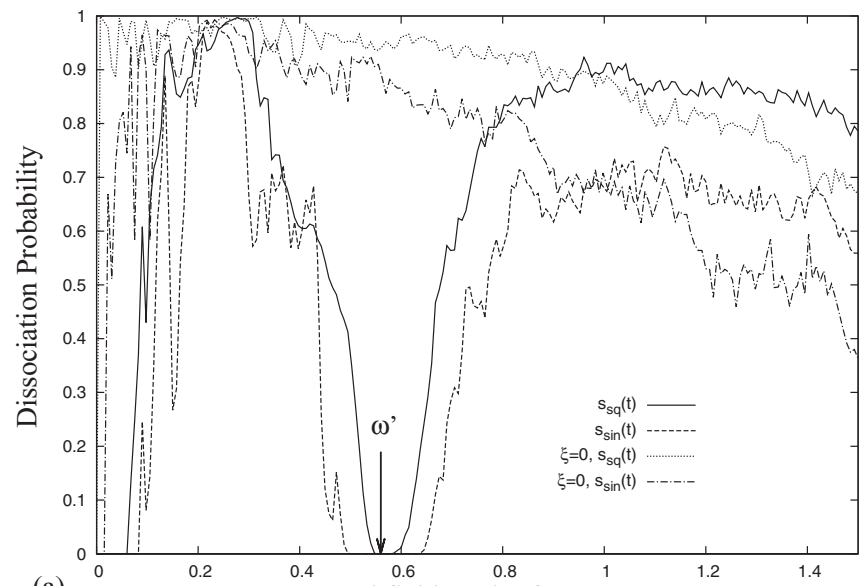

(a) External field carrier frequency $\omega$

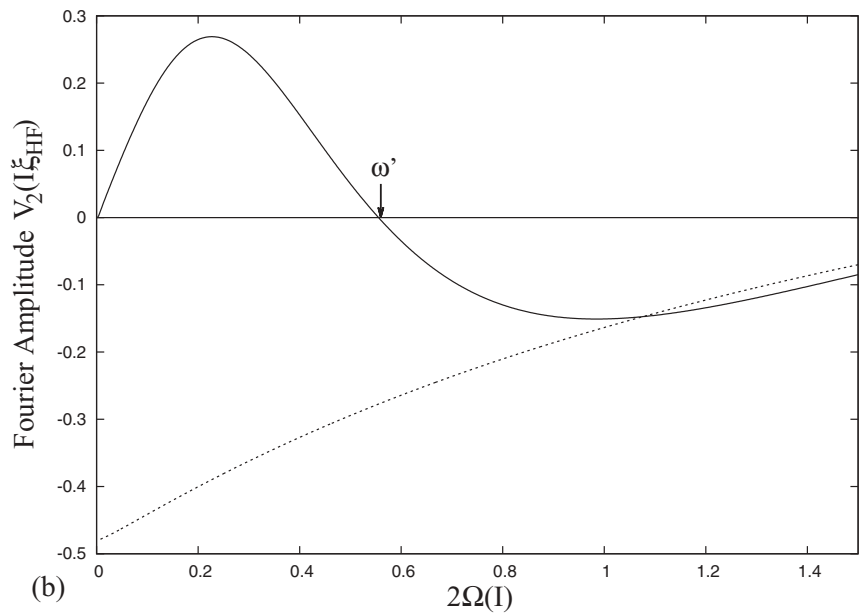

FIG. 4. (a) $P_{D}$ as a function of $\omega$ for square pulses and sinesquared pulses. HF parameters in the solid and dashed curves. $\xi=0$ for the dotted and dot dashed curves. $\epsilon_{0}=0.017$. $I(t=0)=0.7$. The arrow indicates $\omega^{\prime}$ corresponding to the vanishing of $V_{2}\left(I, \xi_{\mathrm{HF}}\right)$, shown in panel (b) (solid line) along with the Fourrier coefficient for $\xi=0$ (dashed line). 
$\epsilon_{0}=0.017$, which is equivalent to $100 \mathrm{MVcm}^{-1}$ or to a peak intensity of $0.26 \mathrm{TWcm}^{-2}$. Whereas for $\xi=0$, the dissociation is considerably high independently of $\omega$, for the HF dipole, the dissociation probability vanishes around $\omega=\omega^{\prime}=0.56$ $\left(\approx 2330 \mathrm{~cm}^{-1}\right)$. Figure $4(\mathrm{~b})$ shows the Fourier coefficient $V_{2}\left(I, \xi_{\mathrm{HF}}\right)$ as a function of the oscillator frequency $2 \Omega(I)$. The dashed line refers to the coefficient for $\xi=0$. The vanishing of the Fourier coefficient corresponds to the resonance condition $\omega^{\prime}=2 \Omega\left(I^{\prime}\right)$. Thus, the suppression of the dissociation of the $\mathrm{HF}$ molecule for $\omega \approx \omega^{\prime}$ can be attributed to the finite range of its permanent dipole moment.

In summary, we have studied the effects of changing the spatial range of the dipole function on the dynamics of the classical driven Morse oscillator. This study has been performed considering an analytical form of the dipole function with adjustable parameters, allowing for the control of the dipole range. An analysis of the nonlinear resonances of the system has shown that for some dipole ranges, the Fourier coefficients associated with the resonances vanish and, correspondingly, the chaotic regions decrease leading to the inhibition of the dissociation. Our nonlinear analysis in the classical counterpart should stimulate corresponding quantum studies in order to verify whether similar dipole-range effects can be observed.

The authors acknowledge support from FAPESP (Grants No. 2010/20276-2 and No. 2010/20683-7). E.F.L. acknowledges support from the Primeiros Projetos program of UNESP.
[1] D. A. Jones and I. C. Percival, J. Phys. B 16, 2981 (1983).

[2] V. Constantoudis and C. A. Nicolaides, Phys. Rev. A 55, 1325 (1997).

[3] R. M. O. Galvão, L. C. M. Miranda, and J. T. Mendonça, J. Phys. B 17, L577 (1984).

[4] J. R. Stine and D. W. Noid, Opt. Commun. 31, 161 (1979).

[5] Y. Gu and J.-M. Yuan, Phys. Rev. A 36, 3788 (1987).

[6] W.-K. Liu, B. Wu, and J.-M. Yuan, Phys. Rev. Lett. 75, 1292 (1995).

[7] B. Wu and W.-K. Liu, Physica A 205, 470 (1994).

[8] V. Constantoudis and C. A. Nicolaides, Phys. Rev. E 64, 056211 (2001).

[9] D. Poppe and J. Korsch, Physica D 24, 367 (1987).

[10] D. Beigie and S. Wiggins, Phys. Rev. A 45, 4803 (1992).

[11] S. Huang, C. Chandre, and T. Uzer, J. Chem. Phys. 128, 174105 (2008).

[12] M. Thachuk and D. M. Wardlaw, J. Chem. Phys. 102, 7462 (1995).

[13] J. Trischka and H. Salwen, J. Chem. Phys. 31, 218 (1959).

[14] L. B. Tran and J. N. Huffaker, J. Chem. Phys. 77, 5624 (1982).

[15] J. Goodisman, J. Chem. Phys. 38, 2597 (1963).

[16] G. Gopakumar, M. Abe, M. Kajita, and M. Hada, Phys. Rev. A 84, 062514 (2011).

[17] R. Guérout, M. Aymar, and O. Dulieu, Phys. Rev. A 82, 042508 (2010).

[18] M. A. Buldakov, V. N. Cherepanov, E. V. Koryukina, and Y. N. Kalugina, J. Phys. B 42, 105102 (2009).
[19] M. A. Buldakov and V. N. Cherepanov, J. Phys. B 37, 3973 (2004).

[20] A. J. Lichtenberg and M. A. Lieberman, Regular and Stochastic Motion (Spring-Verlag, Berlin, 1983).

[21] R. B. Shirts and T. F. Davis, J. Phys. Chem. 88, 4665 (1984).

[22] J. Heagy and J. M. Yuan, Phys. Rev. A 41, 571 (1990).

[23] V. Constantoudis and C. A. Nicolaides, J. Chem. Phys. 122, 084118 (2005).

[24] Z. Wu, Z. Zhu, and C. Zhang, Phys. Rev. E 57, 366 (1998).

[25] K. M. Christoffel and J. M. Bowman, J. Phys. Chem. 85, 2159 (1981).

[26] E. F. de Lima and R. E. de Carvalho, Physica D 241, 1753 (2012).

[27] R. B. Walker and R. K. Preston, J. Chem. Phys. 67, 2017 (1977).

[28] A. Sethi and S. Keshavamurthy, Phys. Rev. A 79, 033416 (2009).

[29] E. F. de Lima and M. A. M. de Aguiar, Phys. Rev. A 77, 033406 (2008).

[30] M. E. Goggin and P. W. Milonni, Phys. Rev. A 37, 796 (1988).

[31] A. Guldberg and G. D. Billing, Chem. Phys. Lett. 186, 229 (1991).

[32] J.-M. Yuan and W.-K. Liu, Phys. Rev. A 57, 1992 (1998).

[33] K. I. Dimitriou, V. Constantoudis, T. Mercouris, Y. Komninos, and C. A. Nicolaides, Phys. Rev. A 76, 033406 (2007).

[34] P. S. Dardi and S. K. Gray, J. Chem. Phys. 80, 4738 (1984).

[35] R. Graham and M. Höhnerbach, Phys. Rev. A 43, 3966 (1991).

[36] R. Graham and M. Höhnerbach, Phys. Rev. A 45, 5078 (1992).

[37] D. Jarukanont, K. Na, and L. E. Reichl, Phys. Rev. A 75, 023403 (2007).

[38] M. V. Korolkov, G. K. Paramonov, and B. Schmidt, J. Chem. Phys. 105, 1862 (1996). 